\title{
Breast Abcess and Tuberculosis and its Diagnostic Challenges: A Two-Year Prospective Study in Karachi, Pakistan
}

\author{
Batool Zehra Asjad ${ }^{1}$, Mir Arsalan Ali ${ }^{2}$, Bushra K. Naeem ${ }^{3}$, Mehmood Khan ${ }^{1}$, Urooj Ahmed Abbasi ${ }^{4}$, \\ Zakia Nehal ${ }^{5}$, Sara Siddiqui ${ }^{5}$ \\ 1. Surgery, Hamdard University Hospital, Karachi, PAK 2. Surgery, Ziauddin University, Karachi, PAK 3. General \\ Surgery, Jinnah Post Graduate Medical College, Karachi, PAK 4. Surgery, Jinnah Postgraduate Medical Center, Karachi, \\ PAK 5. Surgery, Jinnah Postgraduate Medical Centre, Karachi, PAK
}

Corresponding author: Batool Zehra Asjad, drbatoolzehra@hotmail.com

\begin{abstract}
Breast tuberculosis (TB) is rare among extrapulmonary tuberculosis cases, and the diagnosis is usually preceded by a high index of suspicion and findings of granulomatous lesions. We conducted this study to evaluate different clinical presentations of breast TB, including its diagnosis and association with lactation. We also examined the association of breast TB with TB elsewhere in the body and contact history. This prospective, descriptive study was conducted at a tertiary care hospital in Karachi, Pakistan from March 2017 to March 2019. The study population consisted of 100 women of age ranging from 30 to 39 years after 10 patients were lost to follow-up. After providing informed written consent and a histopathology or culture report, participants completed a proforma. We used IBM SPSS Statistics for Windows, Version 22.0 (IBM Corp., Armonk, NY) to analyze the data, which were kept confidential. Twenty-four patients had diabetes, while 36 patients had no comorbidities. The most consistent symptoms were breast pain (98\%), breast lump (89\%), fever (83\%), and discharge (45\%). Of the 100 women, 34 were diagnosed with TB, while 66 women had nonspecific inflammation. Sixty-nine women were lactating, and 31 were nonlactating. Of the 69 lactating women, 21 had been diagnosed with TB while 48 had nonspecific inflammation. TB represents a great burden in underdeveloped countries like Pakistan. Therefore, concerned departments must take necessary action to facilitate early diagnosis and prompt treatment.
\end{abstract}

Received 10/04/2019

Review began 10/08/2019 Review ended 10/11/2019 Published 10/15/2019

๑) Copyright 2019 Asjad et al. This is an open access article distributed under the terms of the Creative Commons Attribution License CC-BY 3.0., which permits unrestricted use, distribution, and reproduction in any medium, provided the original author and source are credited.
Categories: General Surgery, Infectious Disease, Public Health

Keywords: breast abcess, tuberculosis, lactation, extrapulmonary tuberculosis

\section{Introduction}

Tuberculosis (TB) is the found to be one of the persistent human infections and widespread all around the world [1]. It has been well known as a pulmonary disorder but $17.5 \%$ cases also have extrapulmonary manifestation [2]. Breast tuberculosis has been a rare entity constituting only $0.1 \%$ of extrapulmonary tuberculosis [2]. However, in developing countries it accounts for about $3.0 \%$ of breast diseases which were treated surgically [3]. The clinical features of breast tuberculosis vary and lesions may be confused with breast carcinoma or pyogenic abscess [4]. The standard diagnostic methods for the identification and confirmation of Mycobacterium tuberculosis is made either by Ziehl-Neelsen staining or culturing. However, these methods have low sensitivity [4]. Therefore, it is hard to diagnose tuberculosis of the breast and in order to have a definitive diagnosis patients usually undergo numerous investigations and ineffective treatments [4]. Other methods like surgical biopsy and fine needle aspiration cytology (FNAC) may also be inconclusive [5]. In most of the cases, high level of suspicion is required for the diagnosis of breast tuberculosis, which includes presence of langhans giant cells in granulomatous lesion, Mycobacterium tuberculosis in culture and response to antitubercular therapy (ATT) [5]. The objective of the present study is to assess different clinical presentation and diagnosis of breast tuberculosis, it's association with lactation and relation with tuberculosis elsewhere in body or contact history.

\section{Materials And Methods}

This prospective, descriptive type of study was conducted at Hamdard University Hospital Karachi, Pakistan from March 2017 to March 2019. Female patients presenting with breast pain or lump, with or without fever or discharge were included in the study after providing written informed consent. Patients previously operated for breast diseases were excluded from the study population. The study protocol was approved by hospital ethics committee. Data were collected via patient-completed proformas. We also collected data on age, comorbidities, symptoms (such as breast pain, breast lump, fever, and discharge), lactating status, histological analysis, TB contact history, and TB presence elsewhere in the body. Patient data was kept confidential, and IBM SPSS Statistics for Windows, Version 22.0 (IBM Corp., Armonk, NY) was used for data analysis.

\section{Results}




\section{Cureus}

One hundred ten women were initially surveyed; however, 10 patients were lost to follow-up, leaving a total of 100 participants in our study. The patients' age range was 30 to 39 years. Twenty-four patients had diabetes, 36 patients had no comorbidities. Thirty-four patients had been diagnosed with TB, while 66 patients had nonspecific inflammation. Sixty-nine women were lactating, and 31 patients were nonlactating. Of 69 lactating patients, 21 had been diagnosed with TB while 48 patients had nonspecific inflammation. Of the 31 nonlactating patients, 13 were diagnosed with TB while 18 patients had nonspecific inflammation (Table 1).

\begin{tabular}{|l|l|l|l|}
\hline & Tuberculosis & Non-Specific Inflammation & Total patients \\
\hline Lactating & 21 & 48 & 69 \\
Non-Lactating & 13 & 18 & 31 \\
Total patients & 34 & 66 & 100 \\
\hline
\end{tabular}

TABLE 1: Lactation and tuberculosis

Twenty-two patients had a history of TB contact, and of those, 14 patients had breast TB (Table 2).

\begin{tabular}{|c|c|c|c|}
\hline & Tuberculous inflammation & Nonspecific inflammation & Total patients \\
\hline TB contact $+v e$ & 14 & 8 & 22 \\
\hline IB contact -ve & 20 & 58 & 78 \\
\hline Total patients & 34 & 66 & 100 \\
\hline
\end{tabular}

TABLE 2: TB contact history and breast TB

The most common symptoms were breast pain (98\%; Table 3), breast lump (89\%), fever (83\%), and discharge (45\%).

\begin{tabular}{|c|c|c|c|}
\hline & Tuberculous inflammation & Nonspecific inflammation & Total Patients \\
\hline Breast pain present & 33 & 65 & 98 \\
\hline Breast pain absent & 0 & 2 & 2 \\
\hline Total patients & 33 & 67 & 100 \\
\hline
\end{tabular}

TABLE 3: Breast pain and TB

\section{Discussion}

In the underdeveloped world, breast TB accounts for fewer than $1 \%$ of the total breast disease incidence [1]. Breast TB is rare in extrapulmonary TB cases. Baharoon et al reported that in 1829, Sir Astley Cooper reported tuberculous mastitis as the pioneer case and named it "bosom's scrofulous swelling” [1]. Breast TB can be primary (i.e., absent in a different area of the body) or secondary (i.e., present elsewhere in the body), which is somewhat more common than primary [4,6]. Primary breast TB can spread either by abrasions or openings present at the ductules of the nipple [4]. Mirsaeidi et al. reported that the age of patients with breast TB ranges from 20 to 40 years, which was similar to our study range of 30 to 39 years [2]. One study found that in a large number of patients, most breast lesions were abscesses and skin fistulations, most of which were found in young women [3]. Breast TB is more common in women, very rarely involving men. However, a recent study found six of 160 breast TB patients were men [7]. TB is known to spread through contact. In a study by Sreeramareddy et al., $11 \%$ of patients with a history of contact with a known TB case eventually developed extrapulmonary TB [8]. In our study, 22 patients had a history of TB contact, and of 
those, 14 patients had breast TB. Lactation can escalate the vulnerability of the patient for breast TB; $33 \%$ of the women with breast TB in one study were lactating, and in our study, $69 \%$ of the women were lactating [9]. The increased vulnerability from lactation may be due to trauma to the ductules and increased blood supply to the tissue common during lactation, making the tissue more prone to inflammation $[4,7]$. When the TB lesion is irregular, stony hard, and adherent to the skin and underlying structures, it becomes difficult to differentiate this condition from breast cancer [1]. In Ocal et al.'s study, all patients had unilateral involvement of breast $\mathrm{TB}$, which is consistent with our findings. Bilateral involvement of the breast is very infrequent, and according to our results, both breasts have equal tendencies to develop TB [10]. The diagnosis of TB is difficult because the typical findings of matted lymph nodes, several sinuses, ulcers, and breast swelling are absent [5]. The specific investigation for breast TB is Ziehl Neelsen stain or culture [11]. Of the diagnosed cases of breast TB, $12 \%$ are branded with acid-fast, and $25 \%$ are positive for TB bacilli [11]. Many authors consider FNAC as an accurate means of breast TB diagnosis. One study reported $73 \%$ of cases with caseous necrosis and epithelioid giant cells [3]. In another study, red hot tender nodularity of the breast along with the pathological findings of FNAC yielded 100\% accuracy in diagnosing TB, whereas fat necrosis, plasma cell mastitis, and actinomycosis (which resemble the pathological results of breast TB) were not reliable factors for differentiating breast TB from other pathologies [7].

Our study was limited in that no staining tests (e.g., Zehl Nelson or immunohistochemistry) were performed on breast tissue samples due to limited resources. Future studies should involve staining all breast tissue samples with immunohistochemistry by anti-Mycobacterium bovis bacille Calmette-Guérin polyclonal antiserum to improve the diagnostic accuracy in breast TB [12].

\section{Conclusions}

Breast abscess is a very rare presentation of TB. Identifying symptoms and using histopathological examinations early is ideal for diagnosing breast TB. In cases with a high index of suspicion for TB, tissue biopsy should be sent at the time of surgery. TB represents a great burden in underdeveloped countries like Pakistan. Therefore, concerned departments must take necessary action to facilitate early diagnosis and prompt treatment.

\section{Additional Information \\ Disclosures}

Human subjects: Consent was obtained by all participants in this study. Hamdard University Hospital Ethical Board Committee issued approval 001. The said article "Breast Abcess and Tuberculosis, Diagnostic Delima In Underdeveloped Countries, a Two Year Prospective Study in a Cosmopolitan Karachi, Pakistan." We approve the trial to be conducted in presented form. The institutional ethical committee expects to be informed with the progress of study, any revision in the protocol and patient information/informed consent . Animal subjects: All authors have confirmed that this study did not involve animal subjects or tissue. Conflicts of interest: In compliance with the ICMJE uniform disclosure form, all authors declare the following: Payment/services info: All authors have declared that no financial support was received from any organization for the submitted work. Financial relationships: All authors have declared that they have no financial relationships at present or within the previous three years with any organizations that might have an interest in the submitted work. Other relationships: All authors have declared that there are no other relationships or activities that could appear to have influenced the submitted work.

\section{Acknowledgements}

I would like to Thank DR. NADIA NAEEM Ph.D.(Molecular Medicine) Dow University of Health Sciences (DUHS) for her immense help and guidance throughout the writing of this article. She was so kind to had time for this from her busy tough schedule. Her efforts are appreciated.

\section{References}

1. Baharoon S: Tuberculosis of the breast: Analysis of 20 cases and a literature review . Ann Thorac Med. 2008, 3:110. 10.4103/1817-1737.41918

2. 2. Mirsaeidi SM, Masjedi MR, Mansouri SD, Velayati AA.: Tuberculosis of the breast: Report of 4 clinical cases and literature review. EMHJ. 2007, 13:670-676.

3. Da Silva BB, Lopes-Costa PV, Pires CG, Pereira-Filho JD, Dos Santos AR: Tuberculosis of the breast: Analysis of 20 cases and a literature review.. Trans R Soc Trop Med Hyg . 2009, 103:559-63.

4. Brown S, Thekkinkattil DK: Tuberculous cold abscess of breast: An unusual presentation in a male patient . Gland Surg. 2016, 5:361-365.

5. Tauro LF, Martis IS, George C, Kamath A, Lobo G, Hegde BR: Tuberculous mastitis presenting as breast abscess. Oman Med J. 2011, 26:53. 10.5001/omj.2011.14

6. Mallick D, Saha M, Chakrabarti S, Chakrabarty J: Tubercular breast abscess- A diagnostic dilemma. J Nepal Health Res Counc. 2014, 12:138-40.

7. Marinopoulos S, Lourantou D, Gatzionis T, Dimitrakakis C, Papaspyrou I, Antsaklis A: Breast tuberculosis: Diagnosis, management and treatment. Int J Surg Case Rep. 2012, 3:548-50. 10.1016/j.ijscr.2012.07.003

8. Sreeramareddy CT, Panduru KV, Verma SC, Joshi HS, Bates MN: Comparison of pulmonary and extrapulmonary tuberculosis in Nepal: A hospital-based retrospective study. BMC Infect. Dis. 2008, 8:8. 


\section{Cureus}

10.1186/1471-2334-8-8

9. Khanna R, Prasanna GV, Gupta P, Kumar M, Khanna S, Khanna AK: Mammary tuberculosis: Report on 52 cases. Postgrad Med J. 2002, 78:422-4. 10.1136/pmj.78.921.422

10. Ocal K, Dag A, Turkmenoglu O, Kara T, Seyit H, Konca K: Granulomatous mastitis: Clinical, pathological features, and management. Breast J. 2010, 16:176-82. 10.1111/j.1524-4741.2009.00879.x

11. Kakkar S, Kapila K, Singh MK, Verma K: Tuberculosis of the breast. A cytomorphologic study . Acta Cytol. 2000, 44:292-6. 10.1159/000328467

12. Karimi S, Shamaei M, Pourabdollah M, Sadr M, Karbasi M, Kiani A, Bahadori M: Histopathological findings in immunohistological staining of the granulomatous tissue reaction associated with tuberculosis. Tuberc Res Treat. 2014, 2014:858396. 10.1155/2014/858396 